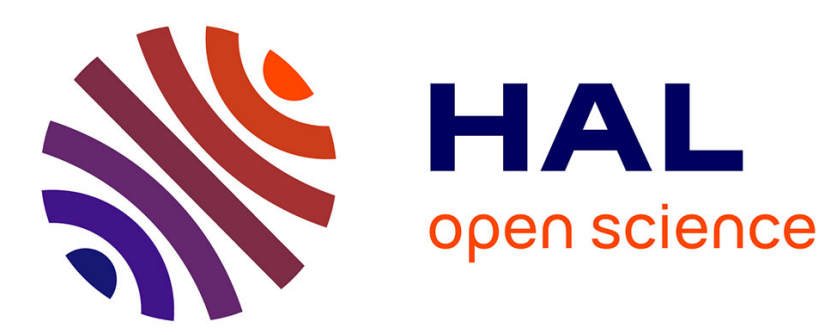

\title{
Computational method for evaluating meteorites as sources of sonic boom
}

Martin Henneton, Philippe Delorme, Olaf Gainville, Christophe Millet, François Coulouvrat

\section{- To cite this version:}

Martin Henneton, Philippe Delorme, Olaf Gainville, Christophe Millet, François Coulouvrat. Computational method for evaluating meteorites as sources of sonic boom. Acoustics 2012, Apr 2012, Nantes, France. hal-00811140

\author{
HAL Id: hal-00811140 \\ https://hal.science/hal-00811140
}

Submitted on 23 Apr 2012

HAL is a multi-disciplinary open access archive for the deposit and dissemination of scientific research documents, whether they are published or not. The documents may come from teaching and research institutions in France or abroad, or from public or private research centers.
L'archive ouverte pluridisciplinaire HAL, est destinée au dépôt et à la diffusion de documents scientifiques de niveau recherche, publiés ou non, émanant des établissements d'enseignement et de recherche français ou étrangers, des laboratoires publics ou privés. 


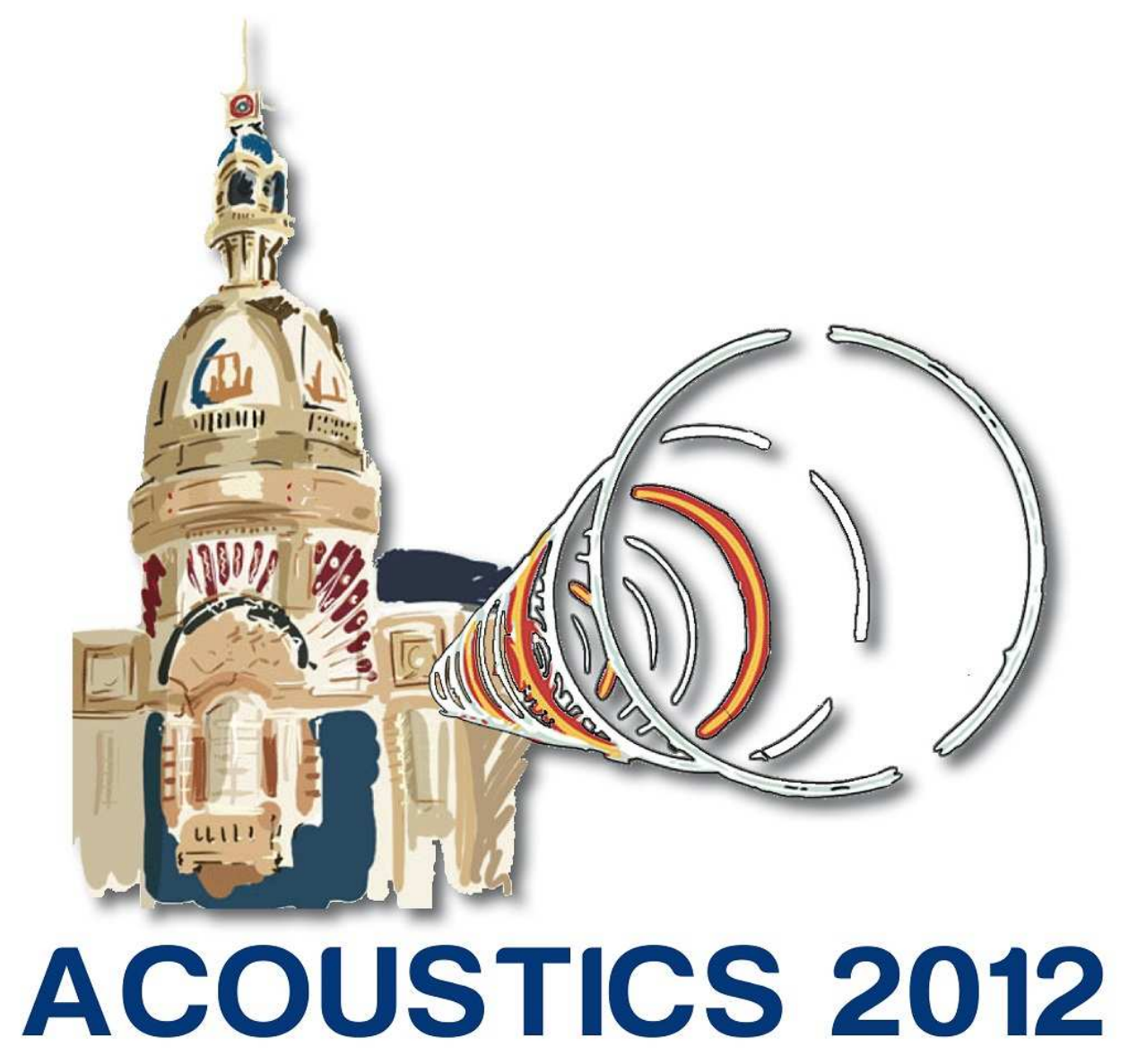

\section{Computational method for evaluating meteorites as sources of sonic boom}

M. Henneton ${ }^{\mathrm{a}}$, P. Delorme ${ }^{\mathrm{a}}$, O. Gainville ${ }^{\mathrm{b}}$, C. Millet $^{\mathrm{b}}$ and F. Coulouvrat ${ }^{\mathrm{c}}$

aONERA, 29 avenue de la Division Leclerc, 92322 Chatillon, France

${ }^{\mathrm{b}}$ CEA, DAM, DIF, F-91297 Arpajon, France

${ }^{c}$ CNRS, Institut Jean Le Rond d'Alembert - UMR CNRS 7190, Université Pierre et Marie

Curie - 4 place Jussieu, 75005 Paris, France

martin.henneton@onera.fr 
Infrasound is one of survey technique monitoring of nuclear explosions. Hence such explosions have to be distinguished from natural sources of infrasounds such as meteorite atmospheric entries. With a view to investigate meteorites as an infrasound source, numerical simulations of Euler equations are performed around a meteorite of diameter one meter entering the atmosphere at Mach 40. This configuration is selected because it corresponds both to a well documented meteorite case and to previous near-field numerical simulations. Because of the very important amount of released energy and to capture the shock, it is necessary to perform the simulations on an adapted mesh. In addition, to obtain the asymptotic weak shock regime and to initialize the nonlinear ray tracing method used to model the long range propagation, simulations have to be performed very far from the meteorite. The output of the CFD simulations are validated by studies on numerical convergence both in the near-field and far-field, and by comparisons with a theoretical model based on the line source assumptions.

\section{Introduction}

Infrasound is one survey technique of the worldwide International Monitoring System in charge of implementing the Comprehensive nuclear Test-Ban Treaty (CTBT) which outlaws nuclear explosions. Its aim is to detect any nuclear test explosion of at least 1 kiloton (1000 tons TNT equivalent). Infrasounds stations of this network frequently detect meteorites atmospherics entries [1-3]. With a view to identify infrasounds sources, it is essential to know if recorded signals correlate with a meteorite entry or other phenomena such as explosions [4], earthquakes [5] or volcanic eruptions [6].

Meteorites as infrasound sources have been first modeled by Lin [7] who considered them as a lineic source of energy with infinite Mach number. The source energy per length unit $E$ emanates from the drag work of the meteorite:

$$
E=\frac{1}{2} \rho_{0} v^{2} c_{x} S,
$$

where $\rho_{0}$ is the atmospheric density, $v$ is the velocity of the meteorite, $c_{x}$ is the drag coefficient and $S$ is the area of the meteorite. The characteristic radius of the cylindrical shock is related to this energy by:

$$
R_{0}=\sqrt{\frac{E}{2 \pi \gamma B p_{0}}},
$$

with $B$ an analytical constant equal to 3.85 for $\gamma=1.40$ and $p_{0}$ the atmospheric pressure. According to Lin's model, pressure, density and velocity distributions are calculated for perfect gases according to a similarity assumption, that is valid only under the strong shock conditions and that generalizes Taylor's self similar solutions [8] to the cylindrical case. As a consequence, it appears that the shock amplitude $\Delta p / p_{0}$ decays as $\left(R / R_{0}\right)^{-2}$ when $\left(R / R_{0}\right) \rightarrow 0$ where $R$ is the radial distance from the meteorite trajectory and $\Delta p$ is the maximum overpressure at the leading shock. Results of this analysis cease to be accurate when the shock strength decays to such an extent that the pressure behind the shock front is no longer greater than the atmospheric pressure. For the long range propagation, Revelle [9] assimilates the pressure signal to a weak shock wave which decays as $\left(R / R_{0}\right)^{-3 / 4},\left(R / R_{0}\right) \rightarrow$ $+\infty$, same laws as the $N$ wave of sonic boom theory [10]. The matching between the two theories is achieved by using the empirical relation proposed by Plooster [11] based on onedimensional numerical simulations of Euler equations in the cylindrical geometry:

$$
\frac{\Delta p}{p_{0}} \approx \frac{2 \gamma}{\gamma+1} \frac{0.4503}{\left(\left(1+4.803\left(\frac{R}{R_{0}}\right)^{2}\right)-1\right)^{3 / 8}} .
$$

The objective of the present work is to go beyond this simplified model and to develop a numerical procedure to evaluate the sonic boom generated by meteorites. The process is similar to the one assessed for computing a sonic boom from supersonic aircraft $[12,13]$. The near-field of the source is computed by CFD Euler numerical simulations. The far-field is evaluated by a nonlinear ray tracing according to Whitham's theory [14]. The main difference between the present work and the classical boom theory is that near-field shocks are much stronger for meteorites, because of the very high Mach number (here we will investigate the case $M=40$ ) and because meteorites are blunt bodies (here we will investigate only the simplify case of spherical meteorites). As a consequence the matching distance between the two procedures is much higher and will be shown here to be several hundreds of thousands times the meteorite diameter.

\section{Shock waves CFD simulations at Mach 40}

\subsection{Selection of the configuration}

The numerical procedure will be assessed on the particular case of a spherical meteorite with a one meter diameter $D$ and with a Mach number equal to 40 . These values have been choosen because they correspond to a well documented meteorite entry $[1,15,16]$ for which the crater is known (near the place of Carancas, Bolivia, 2007) and has been analyzed, and for which infrasound signal have been recorded at $80 \mathrm{~km}$ away from the crater [17]). By estimating the most plausible trajectory of this meteorite, one can estimate the Mach number to be about 40 in the altitude range $40-100 \mathrm{~km}$. At such altitudes, the Knudsen number is sufficiently small so that the atmosphere can be considered as a continuous medium, and the Reynolds number is sufficiently large so that the Euler approximation appears to be correct. Also, at such high altitudes, ablation may be negligible [18] and the meteorite is at constant diameter. Recently, the present case has also been numerically studied by Laurence [19] in order to evaluate the aerodynamic behavior.

\subsection{Presentation of the numerical solver}

The numerical solver chosen in the present work is elsA, the ONERA ${ }^{1}$ software for complex external and internal flow simulations and for multi-disciplinary applications involving aerodynamics [20,21]. Euler equations are solved by a finite volume method on structured meshes with various available schemes such as the van Leer [22], Roe [23] or

\footnotetext{
${ }^{1}$ Office Nationale d'Etudes et de Recherches Aérospatiales.
} 
AUSMP [24] schemes. The selected van Leer scheme turns out to be less diffusive than Roe's one, while the AUSMP method fails at Mach 40. Here we adopt a second order backward Euler method for the pseudo-time integration. Using the meteorite symmetry, simulations are performed with the axisymmetric assumption so that the problem is reduced to a two-dimensional problem in coordinates $(x, R)$, with $x$ is the stream wise coordinate oriented is the direction of the upstream flow, and $R$ the radial coordinate.

\subsection{Mesh description}

Calculations are made with two different meshes. Both of them are divided into several blocks (see figure 1), the first one being centered on the meteorite, and the other ones being aligned with the Mach cone. In the initial mesh, the meshes are approximately of the same size. This initial mesh is used in a preliminary simulation to locate the shock position. The second mesh (called here "optimal mesh") is similar to the first one with the same methodology, but a mesh refinement is applied to all blocks (but the first one) around the head shock position to ensure that this one is well captured (see figure 1) even at very larges distances from the meteorite. This choice allows us to propagate the shock generated by the hypersonic flow around the meteorite to a radial distance of 265 diameters of meteorite, and to a longitudinal distance of 12000 diameters. The total number of points is equal to about 34 millions. Simulations have been performed using MPI parallelization on 32 processors and the total CPU time is 4160 hours.

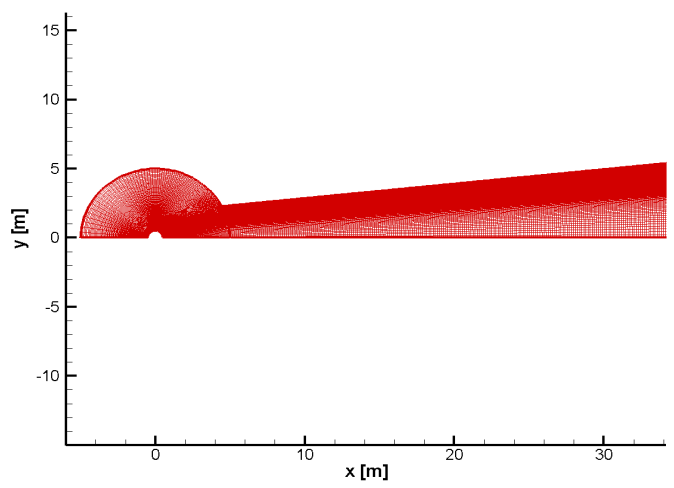

Figure 1: Sketch of the two first blocks of the optimal mesh, with the first one centered on the meteorite, and the second one aligned with the Mach cone and refined around the shock position.

For these calculations, a supersonic input flux is applied on the first left half of the outer boundary of the first block. Axis $R=0$ is defined as a symetrical axis. A wall condition is set on the meteorite surface. Extrapolation conditions [25] are applied to other boundaries.

\subsection{Near-field aerodynamic field analysis}

Before propagating the shock far away from the meteorite, it is necessary to check whether the 2D Euler solver gives the correct behavior for an hypersonic flow around a sphere. To do this, we first compare our near-field simulations around the meteorite to those of Laurence [19]. As visible in figures 2(a) and 2(b) we observe in agreement with this previous work: (1) a detached shock at the stagnation point ahead of the meteorite, (2) a flow separation on the edge of the meteorite, (3) a zone of recirculation behind the sphere and near the axis, (4) a sonic line and a second oblique shock associated to the flow recompression behind the sphere. All these flow features appear in qualitative agreement with [19, 26].
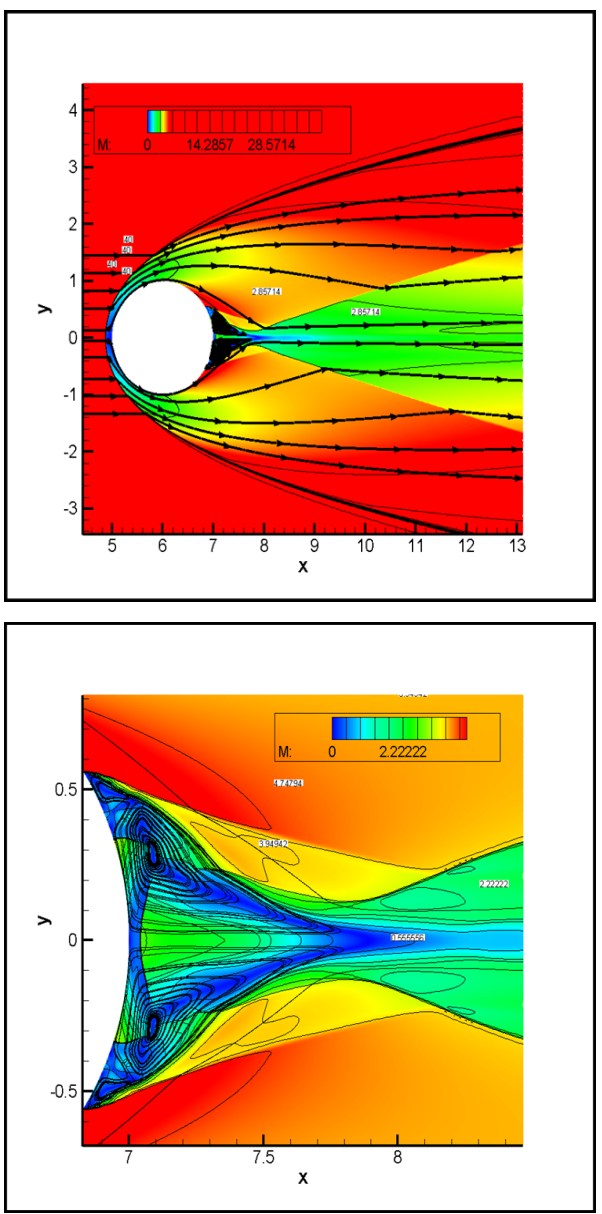

Figure 2: In the top, Mach contours and isobar lines of the aerodynamic near-field flow for a one meter diameter and Mach 40 meteorite (figure 2(a)). In the bottom (figure 2(b)): zoom on the recirculation zone.

\section{Validation of results}

\subsection{Near-field convergence}

Eulerian simulations of shock waves are computed on the two different grids previously introduced (the initial one and the optimal one) to study the mesh convergence. Calculations are made using all mesh points $(1 / 1)$, one point over two (1/2) and one point over four (1/4), in all directions. At the stagnation point (see figure 3(a)), it appears that the convergence of the shock position depends of the number of cells, but the theoretical value of the pressure coefficient $c_{p}=1.839, x=-0.5 \mathrm{~m}$ obtained from the Rankine Hugoniot relations [27] at infinite Mach number is recovered. The convergence of the pressure coefficient on the meteorite surface (see figure 3(b)) appears to be obtained except behind the separation point $(x=0.35 \mathrm{~m})$. Indeed in the recirculation zone the flow is unsteady and strongly turbulent. In this region, convergence can be achieved only by using NavierStokes equations with a turbulent model. The important point 
here is that the flow within that region does not perturb the shock far from the meteorite. Finally, a convergence test is made at $x=10 \mathrm{~m}$ on the intermediate pressure waveform on figure 3(c). One clearly observed the very strong head shock with a pressure ratio $\left(\Delta p / p_{0}\right)$ larger than 20 , followed by the recompression shock. The shock wave obtained by the calculation on the initial mesh (1/1 and 1/4) are compared to the calculation on the shock optimal mesh (1/1 and $1 / 4)$. It appears that the convergence on the value of the shock position is satisfyied for all meshes, but the use of the optimal mesh is necessary to get the correct maximum of the overpressure.

\subsection{Far-field convergence}

The focus of our work is to model the sonic boom generated by the hypersonic entry of meteorites. To match with the sonic boom theory (Whitham [10]), it is necessary to propagate the shock very far from the meteorite. We present on figure 4(a), 4(b) and 4(c) three signals extracted respectively from the initial mesh (1/4) and from the shock adaptated mesh $(1 / 4,1 / 1)$ respectively at $x=2000 \mathrm{~m}, x=5000 \mathrm{~m}$ and $x=6000 \mathrm{~m}$. We can notice that the far field convergence is obtained. A difference of only $9 \%$ is observed between the initial mesh (calculation made with one point over four) and the optimal mesh (calculation made with all points), this last one providing however sharper shocks as expected. We can notice that in the far-field the two shocks have merged. The pressure waveform also shows a negative phase relative to the ambient pressure which is typical for explosion signals $[28,29]$. This kind of behavior is characteristic for the near- to far-field transition [11]. We can also notice that at distances $x$ larger than $5 \mathrm{kms}$ the relative shock amplitude is less than 0.1 , hence within the weak shock acoustic regime.

\section{Comparison with analytical models}

\subsection{Validity of the cylindrical assumption.}

The theoretical model of Lin [7] (near-field) and Revelle [9] (far-field) is based on the assumption of a line source associated with a cylindrical symmetry. We check on the figure 5 the validity of this assumption by comparing the radial velocity $v_{r}$ to the axial one $v_{x}-v_{\infty}$ where the upstream velocity $v_{\infty}=M c_{0}$, with $c_{0}$ the ambient sound speed. Both of these velocities are normalized by the upstream velocity. It clearly appears that in the far-field region the radial velocity is at least two orders of magnitude larger than the axial one, so that the assumption appears to be well satisfied, the difference between the two being of the order of $1 / M$ as could be expected.

\subsection{Near the meteorite: comparison to Lin's model}

As it was emphasis in the introduction, Lin [7] modeled the shock generated by the entry of the meteorite as a line explosion of energy density $E$ given by the work the work of the drag force. We present in figures 6(a), 6(b) and 6(c) some comparisons between our Eulerian Mach 40 simulations (extracted at $x=10 \mathrm{~m}, x=40 \mathrm{~m}$ and $x=100 \mathrm{~m}$ ) and the cylindrical shock wave model of Lin. We can notice two points.
Firstly, the theoretical model overestimates the shock amplitude. Secondly, the numerical simulations show a pressure decay behind the shock faster than the model. As expected, the model based on an ideal line source explosion is not able to describe the second shock due to the geometry of the meteorite. This is clearly showns in $x=10 \mathrm{~m}$ (figure 6(a)).

\subsection{Far-field: comparison with Revelle's model}

We present on figure 7(a) the comparison between Revelle's pressure decay law [9] which is recalled in the introduction (see equation 3 ) and our simulation. The weak shock regime $\Delta p / p_{0}<0.1$ is obtained at the distance $R / R_{0} \approx 7$. In this region, the decay law $\left(R / R_{0}\right)^{-3 / 4}$ is well recovered. In the near-field, the amplitude decay law is approximatively satisfied but the amplitude of the simulation is smaller than the simplified theoretical model. This discrepancy is likely due to the finite geometry of the meteorite with complex flow features (separation point, recirculation zone, recompression shock ...) that cannot be describe by the model. On figure 7(b) is presented the $L_{2}$ relative error between our Mach 40 Euler simulation (on the adaptated grid) and the empirical law of Revelle. In the strong shock region $\left(R / R_{0}<<\right.$ $1)$ the agreement between our simulation and the empirical law is less than $10^{-1}$, while it becomes less than $10^{-5}$ in the weak shock region.In figure 7(c) is given the wave frequency of the shock versus the radial distance. According to Revelle, at $R / R_{0}=10$, the frequency of the wave is equal to $f=\frac{c_{0}}{2.81 R_{0}}$, with $c_{0}$ the speed of sound. Revelle defined a signal characteristic frequency as the inverse of the duration of the compression phase. We can see in figure 7(c) that this frequency calculated by els $A$ is equal to $13.8 \mathrm{~Hz}$ at $R / R_{0}=7.50$, not really far from the theorical value of Revelle which is here equal to $13.1 \mathrm{~Hz}$. Note also that the relation $f \approx\left(R / R_{0}\right)^{1 / 4}$ is well satisfied at distances larger than $2 R_{0}$.

\section{References}

[1] A. Le Pichon. Evidence for a meteoritic origin of the september 15, 2007, Carancas crater. The Meteoritical Society, pages 1797-1810, 2008.

[2] A. Le Pichon, E. Blanc, and A. Hauchecorne. Infrasound Monitoring for Atmospheric Studies. Springer, 2010.

[3] W.N Edwards and al. Infrasonic observations of meteorids: Preliminary results from a coordinated opticalradar-infrasound observing campaign. Earth, Moon, and Planets, 102:221-229, 2008.

[4] O. Gainville, Ph. Blanc-Benon, E. Blanc, R. Roche, C. Millet, F. Le Piver, B. Despres, and P. F. Piserchia. Misty Picture: A Unique Experiment for the Interpretation of the Infrasound Propagation from Large Explosive Sources. Springer, 2009.

[5] J.P Mutschlecner and R. W. Whitaker. Infrasound from earthquakes. Journal of Geophysical Research, 110:1$11,2005$.

[6] J. B. Johnson, R. C. Aster, and P. R. Kyle. Volcanic eruptions observed with infrasound. Geophysical Research Letters, 13:1-4, 2004. 
[7] S.-C. Lin. Cylindrical shock waves produced by instantaneous energy release. Journal of Applied Physics, 25:54-57, 1953.

[8] G. I. Taylor. The formation of a blast wave by a very intense explosion. i. theoretical discussion. Proc. Roy. Soc., A201:159-174, 1950.

[9] D. O. Revelle. On meteor generated infrasound. Journal of Geophysical Research, 81:1217-1229, 1976.

[10] G. B. Whitham. The flow pattern of a supersonic projectile. Comm. on Pure and Applied Mathematics, 5(3):301-348, 1952.

[11] M. N. Plooster. Shock waves from line sources. Physics of fluids, 13:2665-2675, 1970.

[12] R. Marchiano, F. Coulouvrat, and R. Grenon. Numerical simulation of shock wave focusing at fold caustics, with application to sonic boom. Journal of Acoustic Society, 114:1758-1771, 2003.

[13] K. J. Plotkin. State of the art of sonic boom modeling. Journal of the Acoustical Society of America, 1:530 536, 2002.

[14] G. B. Whitham. On the propagation of weak shock waves. Journal of Fluid Mechanics, 1:290-318, 1956.

[15] J. Borovicka and P. Spurny. The Carancas meteorite impact - encounter with a monolithic meteorid. Astronomy and Astrophysics, 485:1-4, 2008.

[16] C. Haynes and C. Millet. A probabilistic study of the carancas meteof fall. Submitted to Geophysical Research Letters, 2011.

[17] P. Brown, D. O. ReVelle, E. A. Silber, W. N. Edwards, S. Arrowsmith, L. E. Jackson Jr, G. Tancreddi, and D. Eaton. Analysis of a crater-formaing meteorite impact in peru. Journal of Geophysical Research, 113:113,2008

[18] Z. Ceplecha. Ablation and shape density coefficients in meteors. Astronomical Institutes of Czechoslovakia, 26:242-248, 1975

[19] S. J. Laurence. Proximal Bodies in Hypersonic Flow. $\mathrm{PhD}$ thesis, California Institute of Technology, 2006.

[20] J. Perraud, J. Cliquet, R. Houdeville, D. Amald, and F. Moens. Transport aircraft three-dimensional high-lift-wing numerical transition prediction. AIAA Aerospace Sciences Meeting and Exhibit $N^{\circ} 17$, 45:1554-1563, 2008.

[21] L. Cambier and J.-P. Veuillot. Status of the elsa cfd software for flow simulation and multidisciplinary applications. AIAA Aerospace Sciences Meeting and Exhibit, 664:1-17, 2008.

[22] B. van Leer. Flux vector splitting for the euler equations. Eighth International conference on numerical methods in fluid dynamics, 170:507-512, 1982.

[23] P. L. Roe. Approximate Riemann solvers, parameter vectors and difference schemes. Journal of Computational Physics, 43:357-372, 1981.
[24] M.-S Liou and C. J. Steffen. A new flux splitting scheme. J. Comput. Phys, 107:23-39, 1993.

[25] M. Lazareff. elsA User's Reference Manual. Office Nationale d'Etudes et de Recherches en Aérospatiales, Décembre 2009.

[26] S. J. Laurence, R. Deiterding, and G. Hornung. Proximal bodies in hypersonic flow. Journal of Fluid Mechanics, 590:209-237, 2007.

[27] P. Lax and B. Wendroff. Systems of conservation laws. Communications on pure and applied mathematics, XIII:217-237, 1960.

[28] G. F. Kinney and K. J. E. Graham. Explosive Shocks in Air. Springer-Verlag, 1985.

[29] P. L. Sachdev. Shock waves and Explosions. Chapman \& Hall / CRC, 2004. 

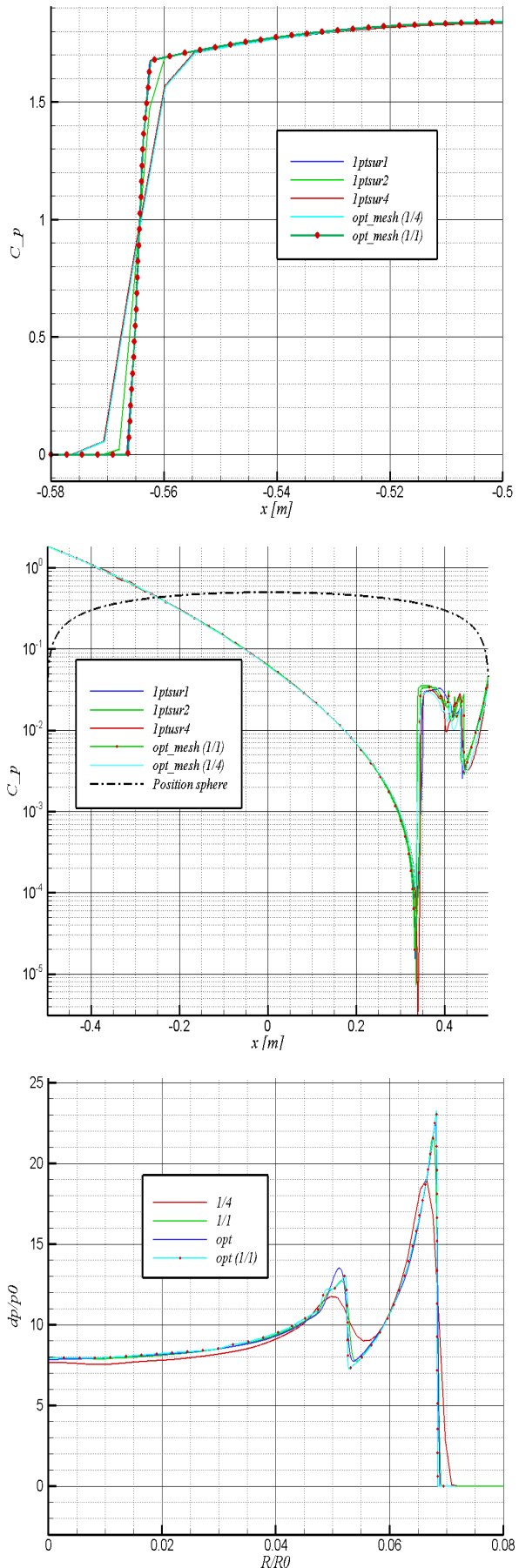

Figure 3: In the top (figure3(a)) the pressure coefficient around the stagnation point of the meteorite obtained from different meshes. The correct value of 1.839 is recovered. In the middle (figure3(b)) pressure coefficient on the meteorite surface. The convergence is obtained except at the separation point. In the bottom (figure3(c)), the pressure waveform extracted at $x=10 \mathrm{~m}$ for different meshes.
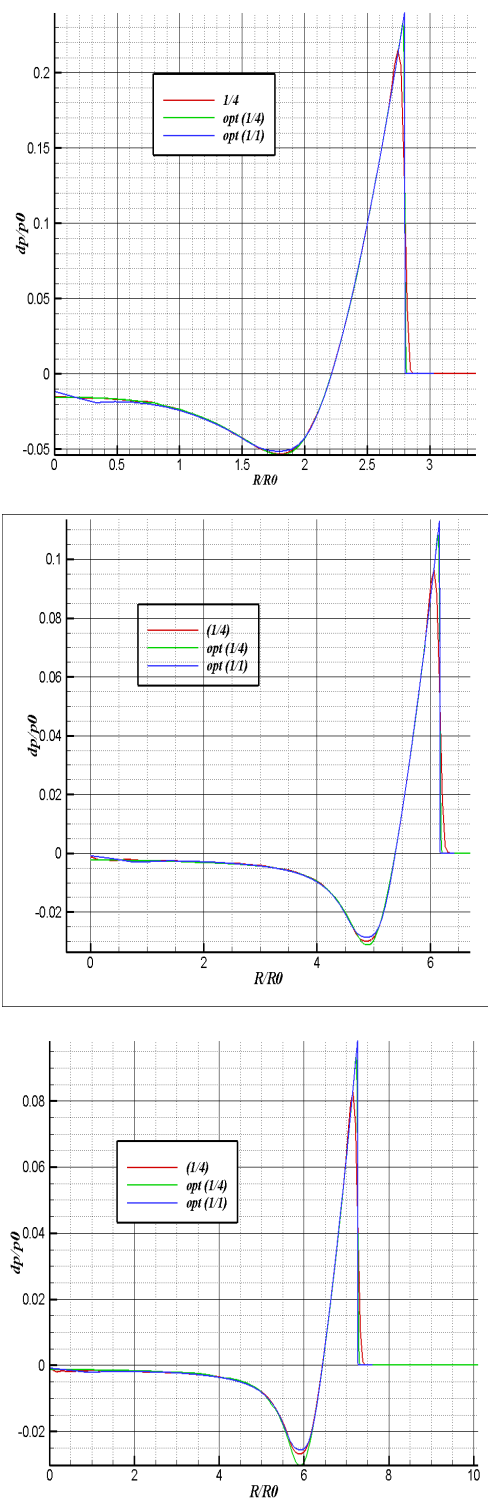

Figure 4: Comparisons of the pressure signals extracted at $x=2 \mathrm{~km}$ (in the top, figure(4(a)), $x=5 \mathrm{~km}$ (in the middle, figure(4(b)) and $x=6 \mathrm{~km}$ (In the bottom, figure(4(c)) for the different meshes.

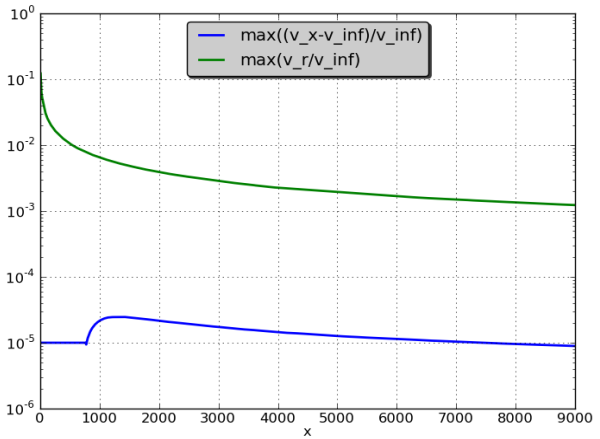

Figure 5: Comparison of the normalized radial $\max \left(\frac{v_{r}}{v_{\infty}}\right)$ and axial $\max \left(\frac{v_{x}-v_{\infty}}{v_{\infty}}\right)$ velocities 

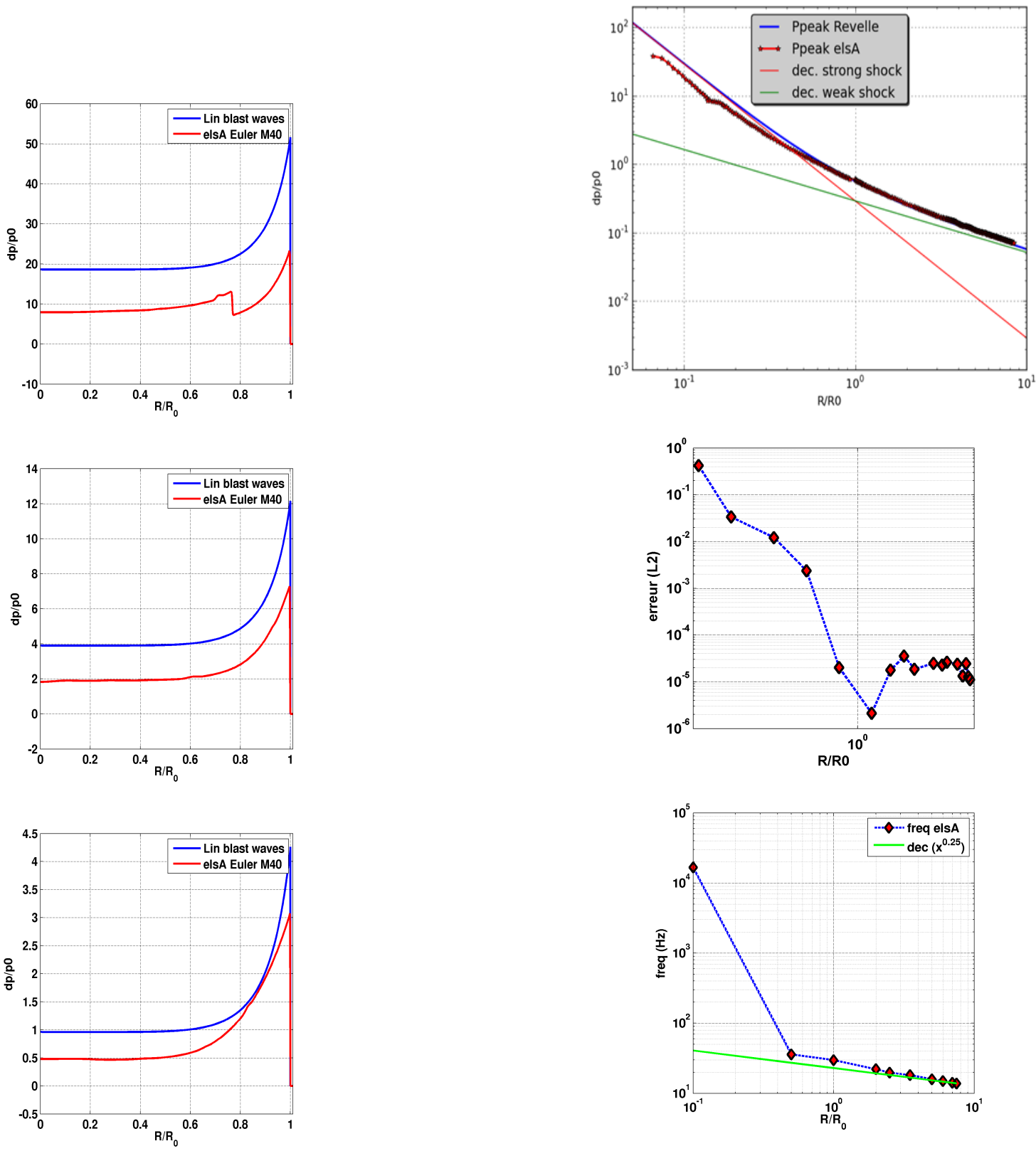

Figure 6: Comparisons between elsA Mach 40 calculations and Lin's model at $x=10 \mathrm{~m}$ (top figure 6(a)), $x=40 \mathrm{~m}$ (middle figure 6(b)) and $x=100 \mathrm{~m}$ (bottom figure 6(c)).

Figure 7: In the top, figure (figure 7(a)): Comparison between elsA Mach 40 simulation, and Revelle's law for the peak overpressure. The two straight lines indicate the near-field $\left((R / R 0)^{-2}\right)$ and far-field $\left((R / R 0)^{-3 / 4}\right)$ theoretical decay laws. In the middle, figure (figure 7(b)): $L 2$ relative error between the simulation and Revelle's model. Bottom figure (figure 5): Calculated shock frequency compared to the weak shock evolution law as $\left(R / R_{0}\right)^{1 / 4}$. 